ARTICLE

\title{
Middle-aged individuals may be in a perpetual state of H3N2 influenza virus susceptibility
}

Sigrid Gouma (10) ', Kangchon Kim², Madison E. Weirick', Megan E. Gumina', Angela Branche ${ }^{3}$, David J. Topham (1) ${ }^{4}$, Emily T. Martin ${ }^{5}$, Arnold S. Monto ${ }^{5}$, Sarah Cobey ${ }^{2} \&$ Scott E. Hensley (i) ${ }^{1 凶}$

Influenza virus exposures in childhood can establish long-lived memory B cell responses that can be recalled later in life. Here, we complete a large serological survey to elucidate the specificity of antibodies against contemporary H3N2 viruses in differently aged individuals who were likely primed with different H3N2 strains in childhood. We find that most humans who were first infected in childhood with H3N2 viral strains from the 1960s and 1970s possess non-neutralizing antibodies against contemporary 3c2.A H3N2 viruses. We find that 3c2.A H3N2 virus infections boost non-neutralizing H3N2 antibodies in middle-aged individuals, potentially leaving many of them in a perpetual state of 3c2.A H3N2 viral susceptibility.

\footnotetext{
${ }^{1}$ Department of Microbiology, Perelman School of Medicine, University of Pennsylvania, Philadelphia, PA 19104, USA. ${ }^{2}$ Department of Ecology \& Evolution, University of Chicago, Chicago, IL 60637, USA. ${ }^{3}$ Division of Infectious Diseases, University of Rochester Medical Center, Rochester, NY 14642, USA.

${ }^{4}$ Department of Medicine and Department of Microbiology and Immunology, David H. Smith Center for Vaccine Biology and Immunology, University of Rochester Medical Center, Rochester, NY 14642, USA. ${ }^{5}$ Department of Epidemiology, University of Michigan School of Public Health, Ann Arbor, MI, USA.

凶email: hensley@pennmedicine.upenn.edu
} 
$\mathrm{M}$ ost humans are infected with influenza viruses by $3-4$ years of age ${ }^{1}$ and have high antibody titers against viral strains encountered early in life ${ }^{2}$. Early childhood influenza exposures can leave lifelong immunological imprints that affect how an individual responds to antigenically distinct viral strains later in life ${ }^{3,4}$. H3N2 influenza viruses began circulating in humans in 1968 and have evolved substantially over the past 51 years 5 . Therefore, an individual's birth year largely predicts which specific type of $\mathrm{H} 3 \mathrm{~N} 2$ virus they first encountered in childhood.

An antigenically drifted descendant of the original 1968 $\mathrm{H} 3 \mathrm{~N} 2$ strain, designated as clade 3c2.A H3N2 viruses, emerged during the 2014-2015 influenza season ${ }^{6}$ and continues to circulate at high levels across most parts of the world ${ }^{7}$. 3c2.A H3N2 viruses are unique because they possess a key glycosylation site that shields HA antigenic site $\mathrm{B}$, which is a major target of neutralizing antibodies ${ }^{8}$. During the 2017-2018 season, 3c2.A H3N2 viruses caused more than 800,000 hospitalizations and more than 60,000 deaths in the United States ${ }^{9}$. Influenza activity is often first detected in children within the community; however, during the severe 2017-2018 influenza season, influenza activity in middle-aged and older adults peaked earlier than in children and young adults ${ }^{10}$. It is surprising that $3 \mathrm{c} 2 . \mathrm{A} \mathrm{H} 3 \mathrm{~N} 2$ viruses dominated the 2017-2018 season in the United States since these viruses also dominated the 2014-2015 and 2016-2017 United States influenza seasons, and therefore many people likely had immunity against these viruses. It is unknown if $3 \mathrm{c} 2$.A viruses were able to circulate widely in 3c2.A H3N2-exposed populations during the 2017-2018 season by acquiring HA and NA substitutions that facilitate antibody escape. Further, it is unknown why these viruses exhibited unusual age-related patterns of circulation during that season and whether or not this was a consequence of different H3N2 exposure histories in different aged individuals.

To determine if HA and/or NA substitutions in 3c2.A H3N2 viruses contributed to the atypical 2017-2018 influenza season in the United States, we completed a large serological survey using samples collected from differently aged individuals in the summer months prior to the 2017-2018 influenza season. We also characterized antibodies in individuals recovering from confirmed 3c2.A H3N2 infections prior to the 2017-2018 influenza season to determine if these antibodies were effective at neutralizing H3N2 viruses that circulated during the 2017-2018 season.

\section{Results}

Serological survey using samples collected prior to the 2017-2018 season. We completed a serological survey using serum samples collected in the summer months prior to the 2017-2018 season from 140 children (age 1-17) at the Children's Hospital of Philadelphia and 212 adults (age 18-90) at the Hospital of the University of Pennsylvania (Supplementary Table 1). First, we used ELISA to measure antibody binding to recombinant HA proteins from a 3c2.A virus from 2014 (herein referred to as 3c2.A) and from a 3c2.A virus from 2018 (herein referred to as $3 \mathrm{c} 2 . \mathrm{A} 2$ ). The $\mathrm{HA}$ of $3 \mathrm{c} 2 . \mathrm{A} 2 \mathrm{H} 3 \mathrm{~N} 2$ viruses from 2018 possesses 3 amino acid substitutions (T131K, R142K, and R261Q) relative to the HA of 3c2.A H3N2 viruses from $2014^{11}$. Most individuals 4 years of age and older possessed 3c2.A and 3c2.A2 HA ELISA-reactive antibodies (Fig. 1a, d). Only 38 of 352 serum samples had no detectable HA-reactive antibodies. Thirty-five of the 38 donors with no detectable HA-reactive antibodies were under 4 years of age, and it is likely that these individuals were not previously exposed to $\mathrm{H} 3 \mathrm{~N} 2$ virus. Importantly, we found no major differences in mean serum antibody binding to 3c2.A HA compared to 3c2.A2 HA (Fig. 1g).
Our ELISAs are designed to detect all HA-binding antibodies, and these assays cannot distinguish between neutralizing and non-neutralizing antibodies. Therefore, we next completed neutralization assays using $3 \mathrm{c} 2 . \mathrm{A}$ and $3 \mathrm{c} 2 . \mathrm{A} 2$ viruses. These assays primarily measure neutralizing $\mathrm{HA}$ antibodies and do not efficiently detect non-neutralizing HA or most NA antibodies. Contrary to what we found in ELISAs, most individuals did not possess high levels of neutralizing antibodies against either 3c2.A or 3c2.A2 viruses (Fig. 1b). Sera from children aged 3-10 years old possessed the highest levels of neutralizing antibodies against these viruses $(p<0.0007$ when compared to all other age groups for both 3c2.A and 3c2.A2 titers, Supplementary Table 2), whereas most middle-aged adults did not have detectable neutralizing antibody titers. Among adults, neutralizing antibody titers were lowest in individuals $\sim 50$ years of age. This is notable since 50-year olds were born in 1967, one year before H3N2 viruses began circulating in humans. This suggests that childhood H3N2 infections in the 1960s and 1970s primed HA antibody responses that are reactive but non-neutralizing against contemporary 3c2.A H3N2 viruses. Neutralizing antibody titers against $3 \mathrm{c} 2 \mathrm{~A} 2$ virus were lower compared to titers against $3 \mathrm{c} 2 \mathrm{~A}$ virus in serum from some individuals, but only 37 individuals (11\%) had a $\geq 4$-fold decrease in neutralizing antibody titer to this virus (Fig. $1 \mathrm{~h}$ and Supplementary Fig. 1). These data suggest that 2017-2018 H3N2 viruses did not possess significant HA antigenic changes relative to 2014-2015 H3N2 viruses for most humans; however, a large fraction of the adult population possessed nonneutralizing antibodies that could bind to the HAs of these viruses but could not prevent virus infection.

We next completed experiments to measure serum antibody binding to NA. Antibodies against NA typically do not neutralize virus infection ${ }^{12}$, so we completed enzyme-linked lectin assays (ELLAs) that measure antibodies that block NA cleavage of sugars on fetuin proteins ${ }^{13}$. Similar to HA neutralizing antibody titers, NA-specific antibody titers were highest in younger individuals and lowest in middle-aged adults (Fig. 1c). Consistent with recent reports 14,15 , we found that some individuals possessed antibodies that reacted to the NA of 3c2.A viruses more efficiently compared to the NA of $3 c 2 . A 2$ viruses (Fig. 2c, f, i). The NA of $3 \mathrm{c} 2 . \mathrm{A} 2$ viruses possesses a new glycosylation site at residue 245 (enabled by S245N + S247T substitutions relative to the NA of $3 \mathrm{c} 2 . \mathrm{A})$ that alters antibody binding ${ }^{14}$. Interestingly, antibodies that were able to antigenically distinguish the 3c2.A and 3c2.A2 NAs were more prevalent in individuals younger than 25 years of age (Fig. 1c, f, i).

It is unclear why many adults born $~ 1968$ possessed low levels of $3 \mathrm{c} 2 . \mathrm{A}$ and $3 \mathrm{c} 2 . \mathrm{A} 2 \mathrm{HA}$ neutralizing antibodies and NA antibodies prior to the 2017-2018 influenza season. It is possible, although unlikely, that $3 c 2$.A viruses did not infect this age group during the previous 2014-2015 and 2016-2017 influenza seasons. An alternative hypothesis is that individuals born $~ 1968$ were infected as much as other adults during the previous $3 \mathrm{c} 2$.A H3N2-dominated seasons but failed to mount neutralizing antibody responses that could confer protection during the 2017-2018 season. To address this possibility, we measured HA and NA antibodies in 14 middle-aged adults (years of birth ranging from 1963-1979) before and after laboratory-confirmed 3c2.A H3N2 influenza virus infection during the 2014-2015 season. These samples were collected as part of the Household Influenza Vaccine Effectiveness (HIVE) study in Ann Arbor, Michigan ${ }^{16}$. Consistent with the hypothesis that $3 \mathrm{c} 2 . \mathrm{A}$ $\mathrm{H} 3 \mathrm{~N} 2$ viruses elicit a non-neutralizing antibody response in middle-aged adults, we found that HA ELISA antibody titers increased in the serum of most individuals 4-8 months after infection (Fig. 2a), but neutralizing antibody titers remained low in the same individuals (Fig. 2b). 3c2.A NA inhibitory antibodies 
3c2.A
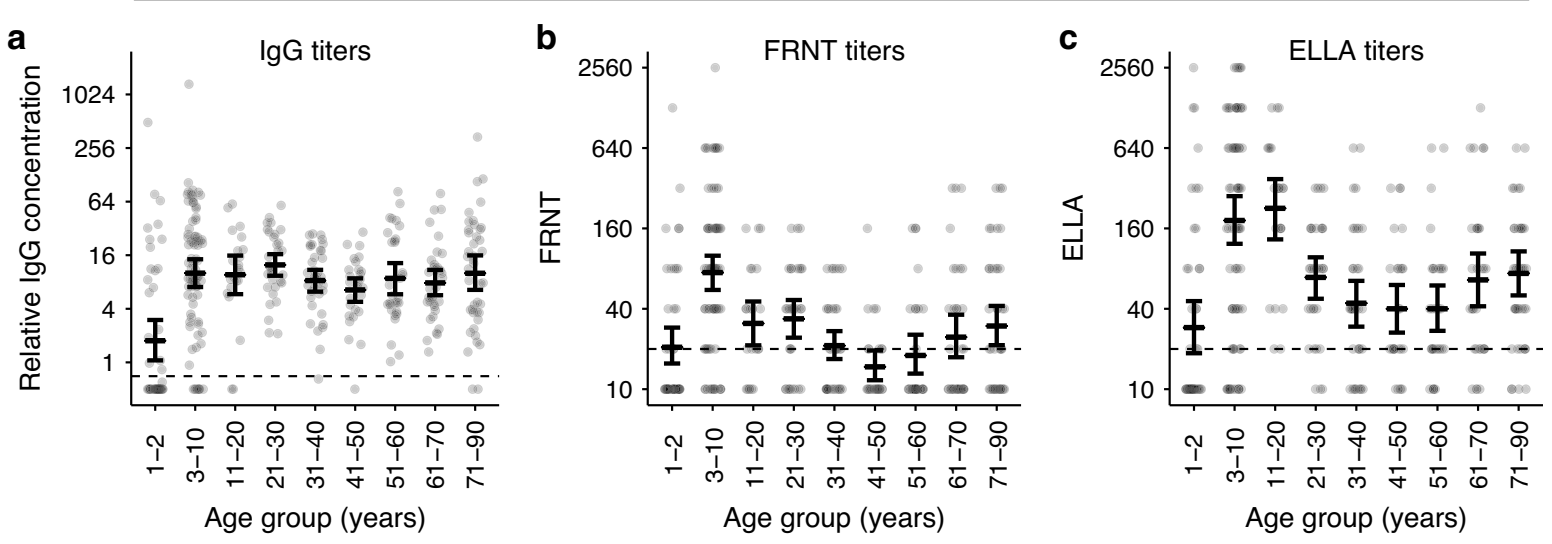

3c2.A2
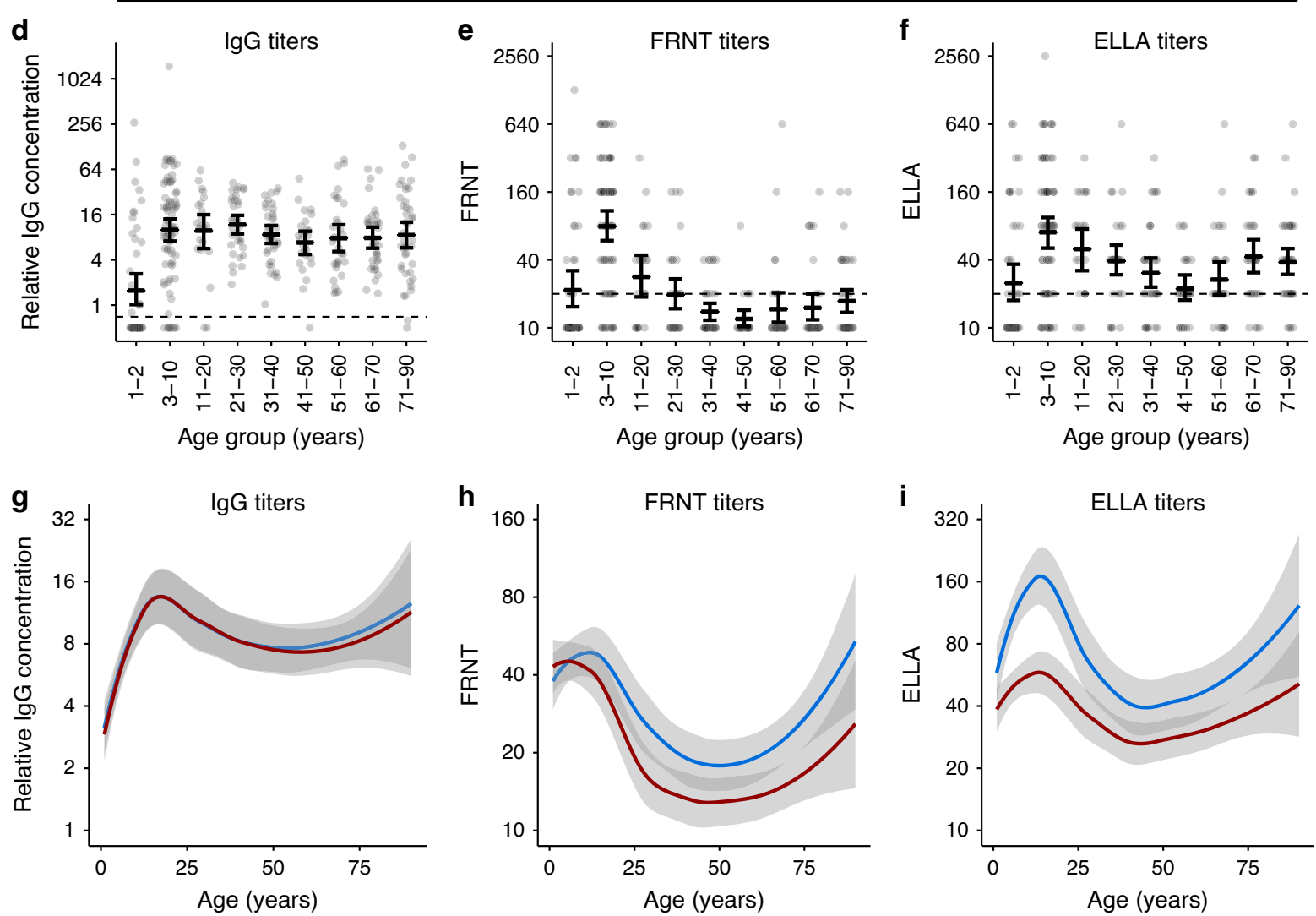

Fig. 1 Antibody titers to contemporary H3N2 viruses in serum collected prior to the 2017-2018 season. ELISAs were completed to quantify the levels of $3 c 2 . A(\mathbf{a})$ and 3c2.A2 (d) HA-specific lgG in serum from different aged humans. FRNTs were completed to quantify the levels of 3c2.A (b) and 3c2.A2 (e) neutralizing antibodies in serum from different aged humans. ELLAs were completed with viruses that had 3c2.A NA (c) and 3c2.A2 NA (f) to quantify NAspecific inhibitory antibodies in serum from different aged humans. For (a-f), the geometric mean titers are shown as cross bars and $95 \%$ confidence intervals as error bars. $\mathbf{g}-\mathbf{i}$ Data from panels (a-f) are summarized. Lines (blue $=3 c 2 . A$; red $=3 c 2 . A 2$ ) are Loess curves showing smoothed geometric mean titers with the $95 \%$ confidence intervals shaded. Dashed lines in (a-f) represent the limit of detection for each assay.

increased in the serum of most infected individuals and these antibodies reacted less well to the antigenically drifted 3c2.A2 NA from 2018 (Fig. 2c). We found similar results when we analyzed serum samples from a separate smaller cohort of individuals $(n=$ 6) collected 28 days post-infection in Rochester, New York during the 2016-2017 season (Fig. 3).

Immune imprinting model. The HAs of $1968 \mathrm{H} 3 \mathrm{~N} 2$ viruses and contemporary $3 \mathrm{c} 2 . \mathrm{A} \mathrm{H} 3 \mathrm{~N} 2$ viruses are dissimilar and most epitopes conserved between these HAs are non-neutralizing (Fig. 4a). We hypothesize that individuals imprinted with H3N2 viruses in childhood in the 1960s and 1970s produce mostly nonneutralizing $\mathrm{HA}$ antibodies against 3c2.A viruses because most of the 3c2.A HA memory B cells in these individuals are those that target non-neutralizing epitopes. We further hypothesize that $3 \mathrm{c} 2$. $\mathrm{A}$ infections in younger individuals do not elicit the same nonneutralizing responses since younger individuals have been primed with viruses more closely related to $3 \mathrm{c} 2 \mathrm{~A}$ (Fig. $4 \mathrm{a}$ ) or they have not yet been primed with $\mathrm{H} 3 \mathrm{~N} 2$ at all. Since $\mathrm{H} 3 \mathrm{~N} 2$ viruses 
a

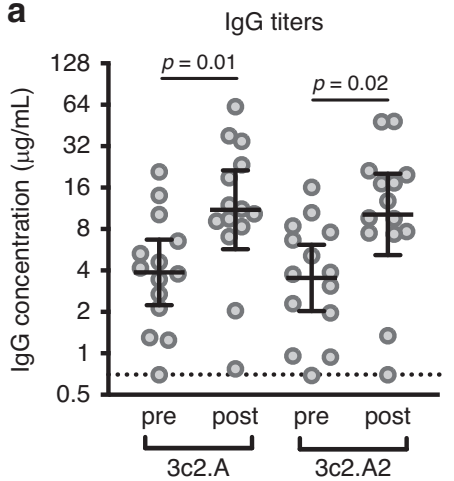

b

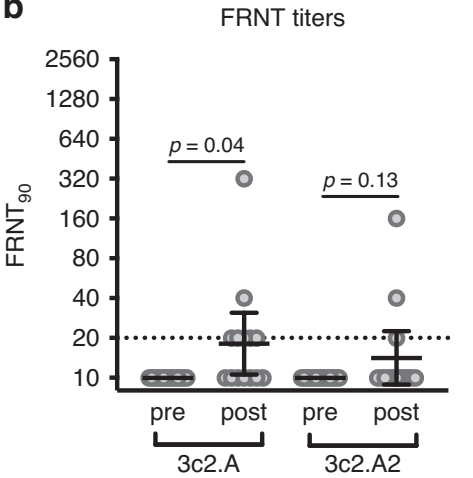

C

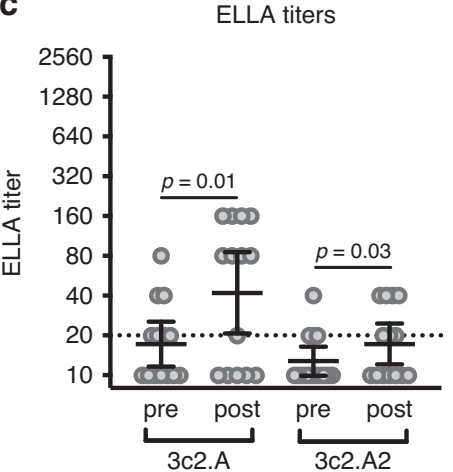

Fig. 2 Antibody responses before and after 3c2. A H3N2 infection during the 2014-2015 influenza season. Serum samples were collected from adults $(n=14$ individuals) before and after laboratory-confirmed 3c2.A H3N2 infection and we quantified (a) HA IgG binding antibodies by ELISAs, (b) neutralizing antibodies by FRNTs, and (c) NA-specific antibodies by ELLAs. Dashed lines represent the limit of detection for each assay. Lines represent the geometric mean titers with $95 \%$ confidence intervals. Two-sided paired $t$ tests were completed on $\log _{2}$-transformed data, and $p$ values are indicated.

a

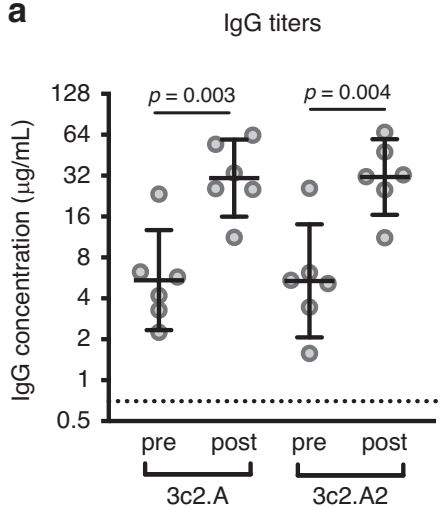

b

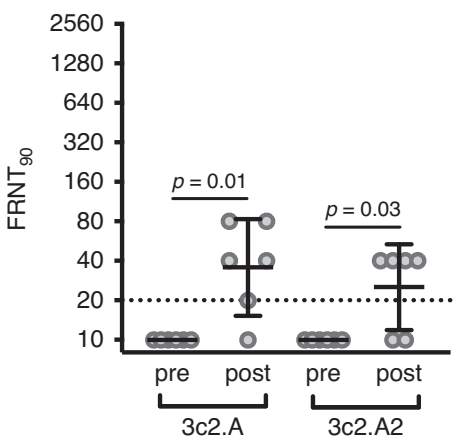

C

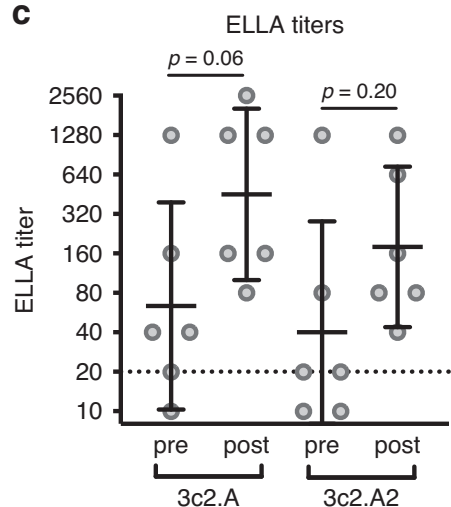

Fig. 3 Antibody responses before and after 3c2.A H3N2 infection during the 2016-2017 influenza season. Serum samples were collected from adults ( $n=6$ individuals) before and after laboratory-confirmed 3c2.A H3N2 infection and we quantified $\mathbf{a}$ HA IgG binding antibodies by ELISAs, $\mathbf{b}$ neutralizing antibodies by FRNTs, and $\mathbf{c}$ NA-specific antibodies by ELLAs. Dashed lines represent the limit of detection for each assay. Lines represent the geometric mean titers with 95\% confidence intervals. Two-sided paired $t$ tests were completed on $\log _{2}$-transformed data, and $p$ values are indicated.

have evolved continuously since 1968, individuals born more recently were likely imprinted with an $\mathrm{H} 3 \mathrm{~N} 2$ strain that shares some neutralizing HA epitopes with $3 \mathrm{c} 2$.A viruses compared to individuals born closer to 1968 . To model this, we calculated the probability of being imprinted with H3N2 for differently aged individuals, taking into account the similarity of the HAs of the imprinting $\mathrm{H} 3 \mathrm{~N} 2$ viral strain with the $\mathrm{HA}$ of $3 \mathrm{c} 2$.A viruses (Fig. 4b). Similarity between the HA of the imprinting $\mathrm{H} 3 \mathrm{~N} 2$ strain and the HA of 3c2.A viruses was determined based on amino acid sequences (see "Methods"). We compared our model predictions with 3c2.A H3N2 neutralizing antibody titers in differently aged individuals (Fig. 4b). Most individuals born prior to the 1960s were not likely imprinted with H3N2 viruses, since these viruses did not start circulating until 1968. Antibody titers against 3c2.A viruses in these older individuals were at similar levels compared to young individuals. Conversely, most individuals born after 1968 were likely imprinted with an H3N2 virus, and this imprinting probability varies for each year of birth after 1977 since H1N1 viruses dominated some influenza seasons after 1977. In our model, being imprinted with an $\mathrm{H} 3 \mathrm{~N} 2$ virus did not correlate with neutralizing antibody titers to $3 \mathrm{c} 2$.A viruses (Supplementary Table 3 ). However, similarity between the HAs of the imprinting $\mathrm{H} 3 \mathrm{~N} 2$ viral strain and the $3 \mathrm{c} 2 . \mathrm{A} \mathrm{H} 3 \mathrm{~N} 2$ viral strain had a positive effect on neutralizing antibody titers $(p<0.001$, Supplementary Table 3). Younger individuals who were imprinted with an $\mathrm{H} 3 \mathrm{~N} 2$ virus with an HA that is more similar to the
HA of 3c2.A H3N2 viruses were more likely to possess neutralizing 3c2.A H3N2 antibodies compared to middle-aged adults who were imprinted with an $\mathrm{H} 3 \mathrm{~N} 2$ virus that has an $\mathrm{HA}$ that is more distant compared to the HA of 3c2.A H3N2 viruses. Taken together, our imprinting model suggests that the specific type of H3N2 virus that an individual is exposed to early in life can affect the specificity of antibody responses against contemporary 3c2.A $\mathrm{H} 3 \mathrm{~N} 2$ viruses.

\section{Discussion}

We propose that middle-aged individuals may be continuously susceptible to $3 \mathrm{c} 2 . \mathrm{A} \mathrm{H} 3 \mathrm{~N} 2$ infection since we found that these viruses preferentially elicit non-neutralizing HA antibody responses in this age group. While non-neutralizing HA and NA antibodies can stop the viral spread and help resolve infections, these antibodies do not typically prevent infections. Although speculative, it is possible that the presence of high levels of nonneutralizing antibodies in middle-aged adults has contributed to the continued persistence of 3c2.A viruses in the human population. Our findings might also relate to the unusual age distribution of H3N2 infections during the 2017-2018 season, in which $\mathrm{H} 3 \mathrm{~N} 2$ activity in middle-aged and older adults peaked earlier compared to children and young adults ${ }^{10}$.

It is interesting that we detected significant NA antigenic change, but only minor HA antigenic change in viral isolates 
a

$+\quad$ Antigenic similarity to 3c2.A2 $\quad-$

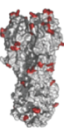

2007

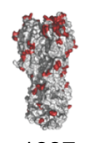

1997

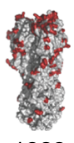

1989

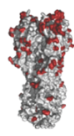

1977

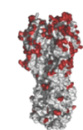

1968 b
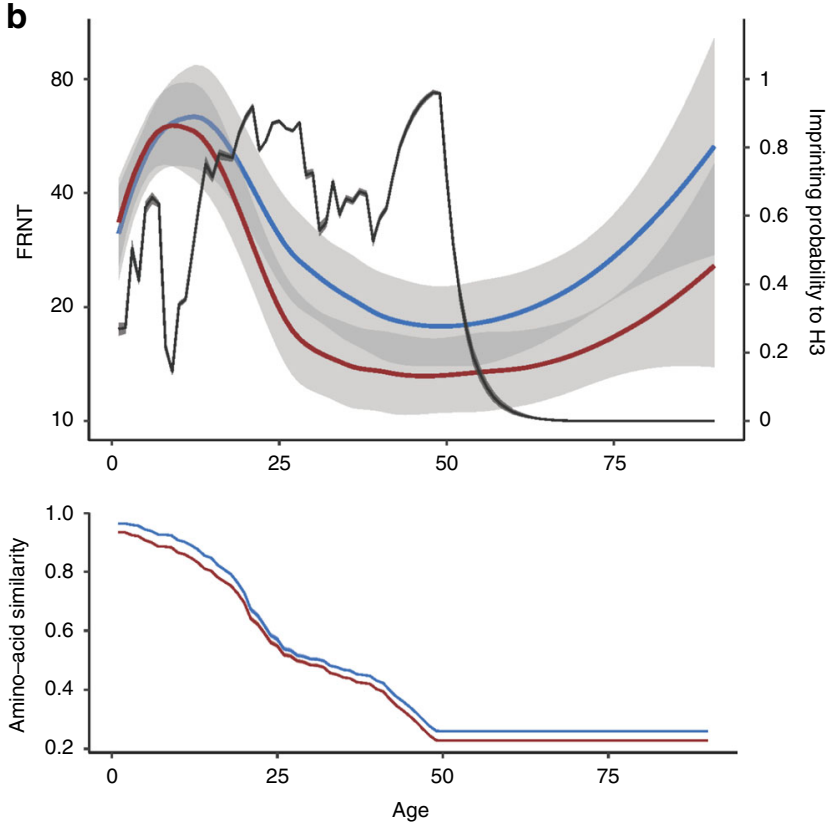

Fig. 4 Early life H3N2 imprinting affects antibodies against 3c2.A

viruses. a $\mathrm{H} 3$ has evolved substantially since 1968 and residue differences between the HA of different H3N2 strains and the 3c2.A2 strain are indicated in red. Accession numbers EF409245 (1968), CY113261 (1977), U97740 (1989), AJ311466 (1997), and EU199250 (2007) were aligned to accession number MH586372 (3c2.A2). b Top, mean FRNT titers to 3c2.A (blue line) and 3c2.A2 (red line) viruses are shown by Loess curves (span $=0.6$ ) with the $95 \% \mathrm{Cls}$ shaded. The probability that an individual was imprinted with $\mathrm{H} 3 \mathrm{~N} 2$ (i.e., their first influenza A virus encounter was H3N2) is indicated with a black line. Bottom, the antigenic similarity between the $\mathrm{HA}$ of an individual's imprinting H3N2 strain and 3c2.A (blue line) and 3c2. A2 (red line).

from the severe 2017-2018 season. We speculate that individuals with non-neutralizing HA antibody responses might rely on NA immunity, and it is possible that this has led to immune pressure on NA and the emergence of viruses with antigenically relevant NA substitutions. We found that NA substitutions in 3c2.A2 viruses from the 2017-2018 season reduced antibody recognition in serum from all age groups but especially from individuals 25years old and younger. Further studies should be designed to tease out the protective role and interplay of HA and NA antibodies during the 2017-2018 season.

Our findings might shed light on the relatively low effectiveness of $3 c 2 . A$ H3N2-based vaccines over the past 3 years ${ }^{17-19}$. Low effectiveness of 3c2.A H3N2-based vaccines is partially due to the inherent difficulties of preparing egg-based 3c2.A H3N2 antigens ${ }^{20}$. 3c2.A H3N2 viruses cannot grow in fertilized chicken eggs without first acquiring an amino acid substitution that abrogates a key glycosylation site in HA antigenic site $\mathrm{B}$, and this egg-adaptation can dramatically alter antigenicity ${ }^{8,21}$. Our study demonstrates that it is difficult to elicit neutralizing antibody responses against $3 \mathrm{c} 2 . \mathrm{A} \mathrm{H} 3 \mathrm{~N} 2$ viruses in some individuals even after natural infection with these viruses. Therefore, irrespective of issues related to $3 \mathrm{c} 2 . \mathrm{A} \mathrm{H} 3 \mathrm{~N} 2$-egg adaptations, it may be inherently difficult to design $3 \mathrm{c} 2 . \mathrm{A} \mathrm{H} 3 \mathrm{~N} 2$ vaccine antigens that are able to elicit neutralizing antibodies in humans that were exposed early in childhood with H3N2 viruses in the 1960s and 1970s.

Future studies should continue to evaluate the specificity of influenza virus antibodies in differently aged individuals. It will be important to continually complete large serological surveys in different aged individuals, including donors from populations with different vaccination rates. A better understanding of immunity within the population and within individuals will likely lead to improved models that are better able to predict the evolutionary trajectories of different influenza virus strains. Large serological studies and basic immunological studies may ultimately shed light on why influenza vaccines elicit variable responses in individuals with different immune histories, while identifying barriers that need to be overcome in order to design better vaccines that are able to elicit protective responses in all age groups.

\section{Methods}

Human serum samples. Serum samples from 140 children (1-17-years old) were collected at the Children's Hospital of Philadelphia (CHOP) between May-August of 2017. Serum samples were originally collected from children for lead testing and leftover de-identified samples were then used for this study. Serum samples from 212 adults (18-90-years old) were collected via the Penn BioBank between May-August of 2017. The Penn BioBank routinely collects serum samples from individuals visiting the University of Pennsylvania Health care system. We did not include samples collected by the Penn BioBank from donors who had a pregnancy reported during the last 9 months, who had a medical history of cancer or organ transplantation, or who had a reported infectious disease within the previous 28 days. We also tested serum collected before and after laboratory-confirmed 3c2. A H3N2 infections during the 2014-2015 and 2016-2017 influenza seasons. The 2014-2015 samples were collected from individuals participating in the HIVE study in Ann Arbor, Michigan ${ }^{16}$. Serum samples from this study were collected 0-3 months before and 4-8 months after laboratory-confirmed 3c2.A H3N2 infection. The 2016-2017 samples were collected from individuals infected in Rochester, NY. Samples from this study were collected at time of laboratoryconfirmed 3c2.A H3N2 infection (referred to in this manuscript as pre-infection samples) and 28 days later (post-infection samples). All studies involving human samples complied with all relevant ethical regulations. Studies were approved by the Institutional Review Boards of the University of Michigan, University of Rochester, and the University of Pennsylvania. Informed consent was obtained from all individuals participating in studies from the University of Michigan and the University of Rochester. Leftover de-identified samples collected at $\mathrm{CHOP}$ were considered exempt from human research (exemption \#4) since the samples were leftover discarded samples that were completely de-identified before our research team received them.

Viruses and recombinant proteins. All viruses used in this study were generated by reverse-genetics ${ }^{22}$. HA and NA genes from A/Colorado/15/2014 (3c.2A) or A/ Pennsylvania/49/2018 (3c.2A2) were cloned into the pHW2000 reverse-genetics plasmid. Viruses were rescued using HA and NA genes from A/Colorado/15/2014 or A/Pennsylvania/49/2018 H3N2 viruses and internal genes from A/Puerto Rico/ 8/1934. For NA experiments, we generated viruses with H6 (A/turkey/Massachusetts/3740/1965) and N2 (from either A/Colorado/15/2014 or A/Pennsylvania/49/ 2018). Viruses were rescued by transfecting reverse-genetics plasmids into cocultures of $293 \mathrm{~T}$ and Madin-Darby Canine Kidney (MDCK)-SIAT1 cells. Transfection supernatants were harvested 3 days after transfection and stored at $-80^{\circ} \mathrm{C}$. $\mathrm{HA}$ and NA genes were sequenced to confirm that additional mutations did not arise during transfection. For the production of recombinant HA proteins, $1 \times 10^{8}$ $293 \mathrm{~F}$ cells were transfected with $70 \mu \mathrm{g}$ codon-optimized A/Colorado/15/2014 HA plasmid in the presence of $10 \mu \mathrm{g}$ NA plasmid using polyethylenimine transfection reagent. Proteins were purified after 3 days and the amount of recombinant HA for both constructs was normalized prior to use in ELISA.

Foci reduction neutralization test (FRNT). Prior to testing in FRNT, serum samples were treated with receptor-destroying enzyme (RDE, Denka Seiken) for 2 $\mathrm{h}$ at $37^{\circ} \mathrm{C}$ and the enzyme was then heat-inactivated at $55^{\circ} \mathrm{C}$ for $30 \mathrm{~min}$. RDEtreated serum samples were serially diluted twofold in Minimum Essential Media (MEM; Gibco). The virus was diluted to a concentration of 300 focus-forming units per well and then incubated with serum samples for $1 \mathrm{~h}$ at room temperature. Confluent monolayers of MDCK-SIAT1 cells were washed twice with MEM and then $100 \mu \mathrm{L}$ of virus-serum mixtures were added to each well in a 96-well plate. Cells were incubated for $1 \mathrm{~h}$ at $37^{\circ} \mathrm{C}$ in $5 \% \mathrm{CO}_{2}$ and then washed with MEM. An 
overlay of $1.25 \%$ Avicel in MEM supplemented with $0.2 \%$ gentamicin and $1 \% 1 \mathrm{M}$ 4-(2-hydroxyethyl)-1-piperazineethanesulfonic acid (HEPES; Gibco) was then added to the cells. After $18 \mathrm{~h}$ of incubation at $37^{\circ} \mathrm{C}$ in $5 \% \mathrm{CO}_{2}$, cells were fixed with $4 \%$ paraformaldehyde for $1 \mathrm{~h}$ at $4{ }^{\circ} \mathrm{C}$. Cells were then lysed with $0.5 \%$ Triton X-100 in PBS for 7 min followed by blocking with 5\% milk in PBS for $1 \mathrm{~h}$ at room temperature. Plates were washed and then $50 \mu \mathrm{L}$ of anti-nucleoprotein monoclonal antibody IC5-1B7 (BEI) 1:5000 diluted in 5\% milk in PBS was added to each well. After incubation for $1 \mathrm{~h}$ at room temperature, plates were washed and $50 \mu \mathrm{L}$ of anti-mouse peroxidase-conjugated secondary antibody (MP Biomedicals) 1:1000 diluted in 5\% milk in PBS was added to each well. Plates were incubated for $1 \mathrm{~h}$ at room temperature, washed, and then 3,3'5,5'-tetramethylbenzidine (TMB) substrate (Kirkegaard \& Perry Laboratories) was added for visualization of the foci. Plates were imaged and foci were quantified using an ELISpot reader. $\mathrm{FRNT}_{90}$ titers are reported as the reciprocal of the highest dilution of sera that reduced the number of foci by at least $90 \%$, relative to control wells that had no serum added. An anti-A/Colorado/15/2014 polyclonal serum in-house control was included in each assay run. Serum samples that did not reduce the number of foci by at least $90 \%$ reduction at a 1:20 serum dilution were assigned an $\mathrm{FRNT}_{90}$ titer of 10 .

Enzyme-linked immunosorbent assay (ELISA). ELISA plates were coated overnight at $4{ }^{\circ} \mathrm{C}$ with recombinant HA protein. The next day, ELISA plates were blocked for $2 \mathrm{~h}$ with PBS containing 3\% bovine serum albumin (BSA; Sigma). Plates were washed three times with PBS containing $0.1 \%$ Tween 20 (PBS-T) and $50 \mu \mathrm{L}$ of diluted serum was added to each well. After $2 \mathrm{~h}$ of incubation, plates were washed three times with PBS-T and then $50 \mu \mathrm{L}$ peroxidase-conjugated anti-human secondary antibody (Jackson ImmunoResearch) diluted in PBS containing 3\% BSA was added to each well. After an hour incubation, plates were washed three times with PBS-T. SureBlue TMB substrate (KPL) was added to develop the plates, and 5 min later $250 \mathrm{mM}$ hydrochloric acid was added to stop the reaction. Plates were read at an optical density (OD) of $450 \mathrm{~nm}$ using the SpectraMax 190 microplate reader (Molecular Devices). A dilution series of monoclonal antibody

041-10047-1C04 starting at $0.5 \mu \mathrm{g} / \mathrm{mL}$ was included on each plate as a control, and $\mathrm{IgG}$ concentrations were calculated based on the titration curve of this antibody ${ }^{23}$. After background subtraction, serum samples that had an IgG concentration below the limit of detection were assigned an IgG concentration of $0.5 \mu \mathrm{g} / \mathrm{mL}$.

Enzyme-linked lectin assay (ELLA). ELLAs were completed to measure NAspecific antibody titers. Microtiter 96-well plates were coated with fetuin diluted in coating solution (KPL) and incubated overnight at $4{ }^{\circ} \mathrm{C}$. Heat-inactivated serum samples were serially diluted twofold in PBS containing $1 \%$ BSA and $0.1 \%$ Tween 20. Coated plates were washed with PBS-T and $50 \mu \mathrm{L}$ of diluted serum was added to each well. Virus was diluted to the appropriate concentration and $50 \mu \mathrm{L}$ of the virus was added to each well. Plates were incubated overnight at $37^{\circ} \mathrm{C}$. The next day, plates were washed with PBS-T and peanut agglutinin conjugated to horseradish peroxidase (PNA-HRP; Sigma) diluted in PBS containing 1\% BSA was added to each well. Plates were incubated for $1 \mathrm{~h}$ at room temperature and then washed with PBS-T. SureBlue TMB substrate (KPL) was added to each well to develop the plates and after 5 min the developing process was stopped with 250 $\mathrm{mM}$ hydrochloric acid. Plates were read at an OD of $450 \mathrm{~nm}$ using the SpectraMax 190 microplate reader (Molecular Devices). After background subtraction, ELLA 50 titers were reported as the reciprocal of the highest dilution of sera that reduced the OD value by at least $50 \%$, relative to control wells that had no serum added. Serum samples that did not show at least $50 \%$ reduction in the OD value at a 1:20 serum dilution were assigned an ELLA $_{50}$ titer of 10.

Modeling. To calculate the probability of being imprinted with H3N2, we followed the approach of Gostic et al. ${ }^{4}$ and estimated the risk of primary H3N2 infection for each birth cohort using surveillance data on the relative intensity of the influenza season, the percentage of influenza A isolates of each subtype that season, and typical attack rates in young children. We defined an influenza season $t$ as from week 40 of year $t-1$ to week 39 of year $t$. The intensity of influenza A in season $t, I_{t}$, was calculated as

$$
I_{t}=\mathrm{ILI}_{t} q_{t}^{\mathrm{A}}
$$

where $\mathrm{ILI}_{t}$ is the mean fraction of all people with influenza-like illness (ILI) in season $t$, and $q_{t}^{\mathrm{A}}$ is the proportion of respiratory specimens testing positive for influenza A out of all respiratory specimens tested in season $t$. For the 1997-1998 to the 2016-2017 seasons, data on ILI and respiratory specimens were obtained from the U.S. Outpatient Influenza-like Illness Surveillance Network (ILINet) and World Health Organization/National Respiratory and Enteric Virus Surveillance System (WHO/NREVSS) Collaborating Labs ${ }^{24}$. For the 1976-1977 to the 1996-1997 seasons, ILI was assumed to be the mean ILI of the above seasons, and the numbers of respiratory specimens were obtained from Thompson et al. ${ }^{25}$. Each $I_{t}$ from the 1976-1977 season to the 2016-2017 season was normalized by dividing by the mean of $I_{t}$ across the seasons. For seasons from 1918-1919 to 1975-1976, we assumed that the intensity of influenza A to be 1 .

We calculated the probability of having the first influenza A exposure to subtype $\mathrm{H} 3 \mathrm{~N} 2$ by taking the approach of Gostic et al. ${ }^{4}$, assigning the probability of infection for naive individuals a value of 0.28 . Then the seasonal attack rate $\alpha$ was calculated by assuming exponential hazard as in Arevalo et al. ${ }^{26}$ :

$$
\alpha=-\log (1-0.28) .
$$

First, we calculated the probability of having the first influenza A exposure in each season for individuals of the same age. We defined individuals of age $a$ to be born in time $y$, from the beginning of July in year $y$-1 to the end of June in year $y$, where year $y$ is 2017 - $a$. The probability of having the first influenza A exposure in season $t$ for individuals born in time $y$ is

$$
p_{y, t}^{\mathrm{A}}=\epsilon_{y, t}^{\mathrm{A}} n_{y, t}^{\mathrm{A}},
$$

where $\epsilon_{y, t}^{\mathrm{A}}$ is the probability of infection with influenza A for naïve individuals born in time $y$ in season $t$, and $n_{y, t}^{\mathrm{A}}$ is the probability of being naïve to influenza $\mathrm{A}$ at the beginning of season $t$. Specifically,

$$
\begin{gathered}
\epsilon_{y, t}^{\mathrm{A}}=1-e^{-I_{t} F_{y, t} \alpha}, \\
n_{y, t}^{\mathrm{A}}= \begin{cases}1, & t=y \\
n_{y, t-1}^{\mathrm{A}}\left(1-\epsilon_{y, t}^{\mathrm{A}}\right), & t>y\end{cases}
\end{gathered}
$$

Here $F_{y, t}$, a fraction of a season $t$ experienced by individuals born in time $y$, was introduced because individuals younger than 6 months of age are assumed not be exposed to influenza due to maternal immunity (see below).

Next, the probability that a cohort born in time $y$ had its first influenza A exposure with subtype $\mathrm{H} 3 \mathrm{~N} 2, P_{y}$, is calculated as

$$
P_{y}=\sum_{t=y}^{y+k} p_{y, t}^{\mathrm{A}} q_{t}^{\mathrm{H} 3},
$$

where $q_{t}^{\mathrm{H} 3}$ is the frequency of $\mathrm{H} 3 \mathrm{~N} 2$ among influenza A cases in season $t$, and $k$ is the maximum age of having a primary influenza $\mathrm{A}$ infection (effectively a numerical correction). Here, we set $k$ to be 20 for individuals of 20 years or older, and to be the age for individuals younger than 20 years. The choice of $k$ minimally affected the value of $P_{y}$.

We calculated the fraction of each season experienced by each cohort following the approach of Arevalo et al. ${ }^{26}$. First, we calculated the fraction of a given influenza season during the week $w$ of season $t, f_{w, t}$ as

$$
f_{w, t}=\frac{\mathrm{ILI}_{w, t} Q_{w, t}}{N_{w, t} \sum_{w \prime=w_{0}}^{w_{f}} \frac{\mathrm{ILI}_{w, t} Q_{w \prime, t}}{N_{w, t}}},
$$

where $\operatorname{ILI}_{w, t}$ is the weighted fraction of patients with ILI in week $w$ of season $t, Q_{w, t}$ is the number of respiratory specimens testing positive to influenza $\mathrm{A}$ in week $w$ of season $t$, and $N_{w, t}$ is the number of specimens tested in week $w$ of season $t$. Parameters $w_{0}$ and $w_{f}$ are the first and the final week of season $t$. For seasons before 1997-1998, weekly data were unavailable. Therefore, for these seasons, we used the mean $f_{w, t}$ of a given week $w$ from the 1997-1998 to the 2017-2018 seasons. We calculated the fraction of an influenza season experienced by an individual born in time $y, F_{y, t}$, using $f_{w, t}$ and the proportion of individuals born in time $y$ that are over 6 months old in week $w$ of season $t, p_{y, w, t}$ :

$$
F_{y, t}=\sum_{w=w_{0}}^{w_{f}} f_{w, t} p_{y, w, t} .
$$

To measure the similarity between an $\mathrm{H} 3$ virus encountered early in life and a test virus, we developed a novel approach of using amino acid similarity between the two viruses. We first calculated the amino acid similarity between $\mathrm{H} 3$ viruses encountered early in life and a test virus, we calculated the probability of having the first influenza H3N2 exposure in season $t$ for individuals born in time $y$ as

$$
p_{y, t}^{\mathrm{H} 3}=\epsilon_{y, t}^{\mathrm{H} 3} n_{y, t}^{\mathrm{H} 3},
$$

where $\epsilon_{y, t}^{\mathrm{H} 3}$ is the probability of $\mathrm{H} 3 \mathrm{~N} 2$ infection for naïve individuals born in time $y$ in season $t$, and $n_{y, t}^{\mathrm{H} 3}$ is the probability of being naïve at the start season $t$, i.e.,

$$
\epsilon_{y, t}^{\mathrm{H} 3}=1-e^{-I_{t} f_{y, t} q_{t}^{\mathrm{H} 3} \alpha}
$$

$$
n_{y, t}^{\mathrm{H} 3}=\left\{\begin{array}{ll}
1, & t=y \\
n_{y, t-1}^{\mathrm{H} 3}\left(1-\epsilon_{y, t-1}^{\mathrm{H} 3}\right), & t>y
\end{array} .\right.
$$

To calculate amino acid similarity, the $\mathrm{H} 3$ sequence samples were downloaded from Global Initiative on Sharing All Influenza Data in August $2018^{27}$. For each season, we resampled 100 samples if the number of available sequences within the season exceeded 100 . The amino acid similarity between $\mathrm{H} 3 \mathrm{~N} 2$ strains circulating in season $t$ and a test virus $v, s_{t, v}$, was calculated as the fraction of shared amino acids in HA head epitopes ${ }^{28}$,

$$
s_{t, v}=\sum_{j=1}^{m_{t}} \sum_{r=1}^{l} \delta_{v, j, r} / \operatorname{lm}_{t},
$$

where $m_{t}$ is the number of $\mathrm{H} 3$ sequence samples collected in season $t, j$ is the index for the sample, $l$ is the number of residues in the H3 head epitopes, $r$ is the index for the residue, and $\delta_{j, r, v}$ is an indicator for the match of the amino acid between a 
test virus $v$ and sample $j$ at residue $r$. The value of $\delta_{j, r, v}$ is 1 if the amino acids match and 0 otherwise. For seasons before 1991-92, where the precise date of sequence isolation was unavailable, we obtained $S_{t, v}$ by calculating the average of $S_{v}$ of year $t$ 1 and year $t$, weighted by the average fraction of sequences from year $t-1$ and from year $t$ for season $t$, calculated from seasons from 1991-1992 to 2016-2017.

Finally, the expected amino acid similarity between the first $\mathrm{H} 3$ virus encountered in life and a test virus $v$ for individuals born in time $y$ is

$$
S_{y, v}=\sum_{t=y}^{y+k} p_{y, t}^{\mathrm{H} 3} s_{t, v} .
$$

We assumed that individuals born before 1967 to have the same amino acid similarity as individuals born in 1967.

To investigate associations between $\mathrm{H} 3$ imprinting, the amino acid similarity between the first $\mathrm{H} 3$ virus encountered in life and a test virus, and titers to the test virus, we performed a linear regression,

$$
H_{i v}=\beta_{1} P_{y_{i}}+\beta_{2} S_{y_{i}, v}+\beta_{3} V_{a_{i}}+c_{v}+c,
$$

where $H_{i, v}$ is individual $i$ 's $\log _{2}$ FRNT titer to test virus $v, P_{y_{i}}$ is the probability of imprinting to $\mathrm{H} 3$ for individual $i$ born in birth time $y, S_{y_{i}, v}$ is the expected amino acid similarity between the first $\mathrm{H} 3$ virus encountered by the birth cohort to which individual $i$ belongs and test virus $v, V_{a_{i}}$ is the vaccination coverage for people the same age as individual $i, c_{v}$ is a virus-specific intercept, and $c$ is constant. Agespecific vaccine coverage from Pennsylvania state for the 2016-17 season was obtained from National Immunization Survey-Flu and Behavioral Risk Factor Surveillance System ${ }^{29}$. We also evaluated models with a linear age effect as

$$
H_{i, v}=\beta_{1} P_{y_{i}}+\beta_{2} S_{y_{i}, v}+\beta_{3} V_{a_{i}}+\beta_{4} a_{i}+c_{v}+c,
$$

where $\beta_{4}$ is the coefficient for age. Finally, we evaluated whether there was support for titer differences in two epidemiologically distinct age groups, children $(<18$ years old) and adults ( $\geq 18$-years old):

$$
H_{i, v}=\beta_{1} P_{y_{i}}+\beta_{2} S_{y_{i}, v}+\beta_{3} V_{a_{i}}+c_{\mathrm{g}_{i}}+c_{v}+c,
$$

where $c_{\mathrm{g}_{i}}$ is the effect for age group $g$ of individual $i$. We used the glm function in $\mathrm{R}$ (version 3.4.2) to fit linear models.

\section{Statistical analysis}

Data are presented as geometric mean antibody levels with 95\% confidence intervals (CI) if not stated otherwise. Analyses were performed using GraphPad Prism (version 7) or R (version 3.4.2). To compare titers between age groups, we performed bootstrap tests according to Hall and Wilson ${ }^{30}$. For a given pair of age groups, we calculate mean titer difference, $\hat{\theta}$, and tested if it is significantly greater than zero. The bootstrap value of $\hat{\theta}, \hat{\theta}^{*}$, is obtained by resampling individuals in each age group with replacement. We obtained the distribution of $\hat{\theta}^{*}$ from 20,000 resamplings. The distribution of $\hat{\theta}$ under a null hypothesis of $\hat{\theta}=0$ is $\hat{\theta}^{*}-\hat{\theta}$ and we calculated $\mathrm{p}$ values from this null distribution. Bootstrapping was performed for every pair of age groups. We then used Bonferroni correction for multiple comparisons by dividing 0.05 by 72 , the number of all pairs of age groups. Therefore, a null hypothesis is rejected if $p<0.0007$. Preand post-infection titers during the 2014-2015 season were $\log _{2}$ transformed and then compared using the paired $t$ - test. $P$ values $<0.05$ were considered as statistically significant.

Reporting summary. Further information on research design is available in the Nature Research Reporting Summary linked to this article.

\section{Data availability}

The sequences used in Fig. 4a are available via GenBank (accession codes EF409245, CY113261, U97740, AJ311466, EU199250 and MH586372). Source data are provided with this paper.

\section{Code availability}

Algorithms are described in detail in the Materials and Methods section. Code is available in the GitHub repository at https://github.com/cobeylab/Flu-Middle-aged.

Received: 9 May 2020; Accepted: 17 August 2020;

Published online: 11 September 2020

\section{References}

1. Bodewes, R. et al. Prevalence of antibodies against seasonal influenza A and B viruses in children in Netherlands. Clin. Vaccin. Immunol. 18, 469-76 (2011).

2. Fonville, J. M. et al. Antibody landscapes after influenza virus infection or vaccination. Science 346, 996-1000 (2014).

3. Francis, T. On the doctrine of original antigenic sin. Proc. Am. Philos. Soc. 104, 572-578 (1960).

4. Gostic, K. M., Ambrose, M., Worobey, M. \& Lloyd-Smith, J. O. Potent protection against $\mathrm{H} 5 \mathrm{~N} 1$ and $\mathrm{H} 7 \mathrm{~N} 9$ influenza via childhood hemagglutinin imprinting. Science 354, 722-726 (2016).

5. Allen, J. D. \& Ross, T. M. H3N2 influenza viruses in humans: Viral mechanisms, evolution, and evaluation. Hum. Vaccin. Immunother. 14, 1840-1847 (2018).

6. Yokoyama, M. et al. Molecular dynamics simulation of the influenza A(H3N2) hemagglutinin trimer reveals the structural basis for adaptive evolution of the recent epidemic clade 3C.2a. Front. Microbiol. 8, 584 (2017).

7. World Health Organization. Recommended composition of influenza virus vaccines for use in the 2019-2020 northern hemisphere influenza season. https:// www.who.int/influenza/vaccines/virus/recommendations/2019_20_north/en/ (2019).

8. Zost, S. J. et al. Contemporary H3N2 influenza viruses have a glycosylation site that alters binding of antibodies elicited by egg-adapted vaccine strains. Proc. Natl Acad. Sci. USA 114, 12578-12583 (2017).

9. Centers for Disease Control and Prevention. Estimated influenza illnesses, medical visits, hospitalizations, and deaths in the United States - 2017-2018 influenza season. https://www.cdc.gov/flu/about/burden/2017-2018.htm.

10. Garten, R. et al. Update: influenza activity in the United States during the 2017-18 season and composition of the 2018-19 influenza vaccine. Mmwr. Morb. Mortal. Wkly. Rep. 67, 634-642 (2018).

11. Neher, R. A. \& Bedford, T. Nextflu: real-time tracking of seasonal influenza virus evolution in humans. Bioinformatics 31, 3546-3548 (2015).

12. Wohlbold, T. \& Krammer, F. In the shadow of hemagglutinin: A growing interest in influenza viral neuraminidase and its role as a vaccine antigen. Viruses 6, 2465-2494 (2014).

13. Couzens, L. et al. An optimized enzyme-linked lectin assay to measure influenza A virus neuraminidase inhibition antibody titers in human sera. J. Virol. Methods 210, 7-14 (2014).

14. Wan, $\mathrm{H}$. et al. The neuraminidase of $\mathrm{A}(\mathrm{H} 3 \mathrm{~N} 2)$ influenza viruses circulating since 2016 is antigenically distinct from the A/Hong Kong/4801/2014 vaccine strain. Nat. Microbiol. 4, 2216-2225 (2019)

15. Powell, H. \& Pekosz, A. Neuraminidase antigenic drift of H3N2 clade 3c.2a viruses alters virus replication, enzymatic activity and inhibitory antibody binding. PLoS Pathog. 16, e1008411 (2020).

16. Malosh, R. et al. Factors associated with influenza vaccine receipt in community dwelling adults and their children. Vaccine 32, 1841-1847 (2014).

17. Flannery, B. et al. Influenza vaccine effectiveness in the United States during the 2016-2017 season. Clin. Infect. Dis. 68, 1798-1806 (2019).

18. Rolfes, M. A. et al. Effects of influenza vaccination in the United States during the 2017-2018 influenza season. Clin. Infect. Dis. 69, 1845-1853 (2019).

19. Doyle, J. D. et al. Interim estimates of 2018-19 seasonal influenza vaccine effectiveness - United States, February 2019. Mmwr. Morb. Mortal. Wkly. Rep. 68, 135-139 (2019).

20. Levine, M. Z. et al. Antibodies against egg- and cell-grown influenza A(H3N2) viruses in adults hospitalized during the 2017-2018 season. J. Infect. Dis. 219, 1904-1912 (2019).

21. Lin, Y. et al. The characteristics and antigenic properties of recently emerged subclade 3C.3a and 3C.2a human influenza A(H3N2) viruses passaged in MDCK cells. Influenza Other Respi. Viruses 11, 263-274 (2017).

22. Chambers, B. S., Parkhouse, K., Ross, T. M., Alby, K. \& Hensley, S. E. Identification of hemagglutinin residues responsible for H3N2 antigenic drift during the 2014-2015 influenza season. Cell Rep. 12, 1-6 (2015).

23. Henry Dunand, C. J. et al. Preexisting human antibodies neutralize recently emerged H7N9 influenza strains. J. Clin. Invest. 125, 1255-68 (2015).

24. Centers for Disease Control and Prevention. FluView national, regional, and state level outpatient illness and viral surveillance. https:/gis.cdc.gov/grasp/ fluview/fluportaldashboard.html (2018).

25. Thompson, W. W. et al. Mortality associated with influenza and respiratory syncytial virus in the United States. JAMA 289, 179 (2003).

26. Arevalo, P., McLean, H. Q., Belongia, E. A. \& Cobey, S. Earliest infections predict the age distribution of seasonal influenza A cases. Elife 9, e50060 (2020).

27. Shu, Y. \& McCauley, J. GISAID: Global initiative on sharing all influenza data - from vision to reality. Eur. Surveill. 22, 30494 (2017).

28. Shih, A. C.-C., Hsiao, T.-C., Ho, M.-S. \& Li, W.-H. Simultaneous amino acid substitutions at antigenic sites drive influenza A hemagglutinin evolution. Proc. Natl Acad. Sci. USA 104, 6283-8 (2007).

29. Centers for Disease Control and Prevention. FluVaxView. https://www.cdc. gov/flu/fluvaxview/local-areas-estimates-2016-17.htm (2018). 
30. Hall, P. \& Wilson, S. R. Two guidelines for bootstrap hypothesis testing. Biometrics 47, 757 (1991).

\section{Acknowledgements}

This work was supported by the National Institute of Allergy and Infectious Diseases (1R01AI113047, S.E.H.; 1R01AI108686, S.E.H.; 1R01AI097150, A.S.M.; CEIRS HHSN272201400005C, S.E.H., S.C., E.T.M., A.S.M., A.B., D.J.T.) and Center for Disease Control (U01IP000474, A.S.M.). Scott E. Hensley holds an Investigators in the Pathogenesis of Infectious Disease Awards from the Burroughs Wellcome Fund.

\section{Author contributions}

S.G., M.W., and M.G. completed experiments and analyzed data. K.K. and S.C. analyzed data and completed modeling studies. A.B., D.J.T., E.T.M., and A.S.M. provided serum samples from individuals before and after PCR-confirmed H3N2 infection. S.E.H. conceived the project and supervised the work. S.G. and S.E.H. wrote the manuscript with input from all authors.

\section{Competing interests}

S.E.H. has received consultancy fee from Sanofi Pasteur, Lumen, Novavax, and Merck for work unrelated to this report. A.S.M. has received consultancy fees from Sanofi Pasteur, Seqirus, and Novavax for work unrelated to this report. A.B. reports have received consultancy fees from GSK and Merck and grant support from Merck, Pfizer, and Janssen for work unrelated to this report. All other authors report no potential conflicts.

\section{Additional information}

Supplementary information is available for this paper at https://doi.org/10.1038/s41467020-18465-x.

Correspondence and requests for materials should be addressed to S.E.H.

Peer review information Nature Communications thanks the anonymous reviewers for their contribution to the peer review of this work.

Reprints and permission information is available at http://www.nature.com/reprints

Publisher's note Springer Nature remains neutral with regard to jurisdictional claims in published maps and institutional affiliations.

(c) (i) Open Access This article is licensed under a Creative Commons Attribution 4.0 International License, which permits use, sharing, adaptation, distribution and reproduction in any medium or format, as long as you give appropriate credit to the original author(s) and the source, provide a link to the Creative Commons license, and indicate if changes were made. The images or other third party material in this article are included in the article's Creative Commons license, unless indicated otherwise in a credit line to the material. If material is not included in the article's Creative Commons license and your intended use is not permitted by statutory regulation or exceeds the permitted use, you will need to obtain permission directly from the copyright holder. To view a copy of this license, visit http://creativecommons.org/ licenses/by/4.0/.

(C) The Author(s) 2020 